\title{
Structural Correspondence of Solution, Liquid Crystal, and Crystalline Phases of the Chromonic Mesogen Sunset Yellow
}

\author{
Wenchang $\mathrm{Xiao}^{\dagger}$, Chunhua $\mathrm{Hu}^{\dagger}$, Damien J. Carter ${ }^{\ddagger}$, Shane Nichols ${ }^{\dagger}$, Michael D. Ward ${ }^{\dagger}$, Paolo \\ Raiteri $^{\ddagger^{*}}$, Andrew L. Rohl ${ }^{\ddagger^{*}}$, Bart Kahr ${ }^{\dagger^{*}}$ \\ ${ }^{\dagger}$ Department of Chemistry and Molecular Design Institute, 100 Washington Square East, Silver Center, Room 1001, \\ New York University, New York, NY 10003-6688, United States \\ ${ }^{\ddagger}$ Nanochemistry Research Institute and Department of Chemistry, Curtin University, P.O. Box U1987, Perth, \\ Western Australia 6845, Australia
}

\begin{abstract}
The azo dye, sunset yellow, is a prototypical, chromonic liquid crystal in which assembly in aqueous solution at high volume fraction leads to lyotropic mesophases with a "package of properties distinct in almost every aspect" (Lydon, J. Curr. Opin. Colloid Interfac. Sci. 2004, 8, 480). In particular, the isotropic to nematic transition in such phases, the consequence of stacking of dye molecules in chains, is difficult to bring into correspondence with athermal theories for rigid rods as well as modifications that consider chain interactions with one another. Chromonic mesogens, small molecules that stack to form lyotropic liquid crystals, prompt structural questions that have yet to be answered; a full understanding of structure should inform colligative properties. Herein, the single crystal structure of a guanidinium salt of the sunset yellow dianion, a known chromonic mesogen, is reported. The compound crystallizes as a dihydrate, tetrahydrofuran solvate in the orthorhombic space group Pnna, with $a=6.8426(5) \AA, b=$ 20.048(1) $\AA, c=21.466(2) \AA$. The sunset yellow molecules, point group approximately $C_{s}$, are disordered about a crystallographic diad axis. The structure is informative because pairwise interactions in the disordered crystal structure show a remarkable correspondence with the stereochemistry of sunset yellow molecules in solution and in the liquid crystal phase. The solution structure is here simulated by the combination of molecular dynamics, metadynamics, and quantum chemical computations. The comparable disorder in the fluid and solid states suggests the possibility that stacked aggregates adhere to growing crystals intact. Computations evaluated proposals that stacking faults and branching points lower the X-ray correlation lengths while preserving extended structures. Evidence is found for stacking faults but not branches. The solution stereochemistry and stereodynamics has implications for the geometry of long rods, for which understanding is a prerequisite for reckoning properties of vexing chromonic mesophases.
\end{abstract}




\section{- INTRODUCTION}

A class of mesophases discovered a century ago ${ }^{1}$ has reemerged in recent years, the so-called lyotropic chromonic liquid crystal (LCLC) ${ }^{2,3,4,5,6,7}$ LCLCs are aqueous phases of supramolecular assemblies of small molecules, often sulfonated dyes. Chromonic mesogens may contain other hydrophilic groups on their perimeters such as carboxylates, as in the case of the anti-asthmatic drug sodium cromoglycate, ${ }^{8,9,10,11}$ the compound whose name is given to this class of compounds, ${ }^{12}$ and whose study brought LCLCs to the attention of contemporary researchers. Unlike typical lyotropic liquid crystal mesogens, the components of LCLCs lack long chains and are not micelle-forming amphiphiles. LCLCs have been used as polarizers, ${ }^{13}$ display components, ${ }^{14}$ and sensors, ${ }^{15}$ but the detailed structures of chromonic aggregates are still largely unknown. ${ }^{5}$

One of the most well studied LCLCs is formed from concentrated solutions of sunset yellow FCF, a food coloring (SSY, disodium 6-hydroxy-5-[(4-sulfophenyl)azo]-2-naphthalenesulfonate, also known as orange yellow S, FD\&C yellow 6, edicol, or as CAS 2783-94-0, Colour Index No. $\left.15985^{16}\right) .{ }^{17,18,19,20,21,22,23,24,25,26} \mathrm{SSY}$ is a disulfonated azo dye at neutral $\mathrm{pH}$. SSY aggregates in aqueous solution, eventually forming a nematic, lyotropic LCLC phase above $\sim 30 \mathrm{wt} \%$ and a hexagonal, columnar phase above $\sim 40 \mathrm{wt} \%$. A variety of analytical methods have been trained on SSY mesophases with the aim of establishing the supramolecular structure of aggregates. Single crystals of SSY have not been reported and, to date, to the best of our knowledge only a few LCLC-forming compounds afford single crystal structures that would provide direct evidence of self-association geometries (cromoglycate (27,28,29 $^{2}$ and a perylenemonoimide ${ }^{30}$ ).

Our long-standing interest in dyeing crystals has led us to generate many mixed crystals containing sulfonated dyes. ${ }^{31,32,33,34} \mathrm{SSY}$ in micromolar concentrations had earlier been studied in crystals of $\mathrm{K}_{2} \mathrm{SO}_{4}{ }^{35,36}$ and by our group ${ }^{37,38}$ and others in $\mathrm{KH}_{2} \mathrm{PO}_{4}{ }^{39,40,41}$ These mixed crystals grow at solution concentrations of SSY known to favor the formation of dimers and trimers, on their way to chromonic aggregates. Understanding SSY assembly is essential for understanding the structure of these mixed crystals. Moreover, the associations of sulfonated dyes are not only of interest in the dyeing of crystals but also in the dyeing of tissues. ${ }^{42}$ For instance, histologists have recognized that Congo red clusters are the active agents in the staining of amyloid plaques associated with neurodegenerative disorders ${ }^{43,44,45,46}$ while other self-associating azo dyes inhibit HIV protease and reverse transcriptase. ${ }^{47,48}$ Therefore, the supramolecular chemistry of SSY and congeners has relevance in a variety of areas of contemporary chemistry.

Herein, we report a single crystal structure of SSY with guanidinium counter ions $\left(\left(\mathrm{CH}_{6} \mathrm{~N}_{3}\right)_{2}^{+} \mathrm{SSY}^{2-}\right.$ or simply $\mathrm{G}_{2} \mathrm{SSY}$ ). Although the structure is disordered, rather than being confounding the disorder clarifies, and provides experimental evidence of the stereochemical complexity of proto-chromonic stacks. ${ }^{49}$ 
Recently, a molecular dynamics (MD) simulation study of SSY in water provided vivid insights into some aspects of its intermolecular association. ${ }^{49}$ In order to achieve a fuller correspondence between this view of SSY assembly and the stereochemical implications of the crystallographic packing, we extended the MD simulations with modifications to the potentials while providing supplementary quantum chemical computations of dimer structures and stereochemistry in vacuo using a vdW-aware DFT functional. Ultimately, the potential energy surfaces for interacting dimers were computed with the metadynamics enhanced sampling technique. ${ }^{50}$

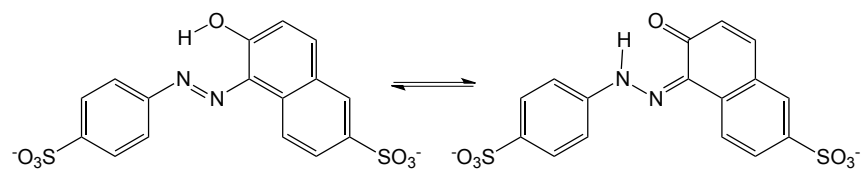

Scheme 1. Chemical structures of sunset yellow (SSY) tautomers

\section{- EXPERIMENTS AND RESULTS}

Crystal Growth. Guanidinium tetrafluoroborate, $\mathrm{GBF}_{4}$, was prepared from $10 \mathrm{~g}$ of $\mathrm{G}_{2} \mathrm{CO}_{3}$ (Sigma) and $14 \mathrm{~mL}$ of $\mathrm{HBF}_{4}\left(48 \%\right.$ in $\mathrm{H}_{2} \mathrm{O}$, Sigma) mixed and stirred for 5 hours, after which all the solvent was removed by rotary evaporation to yield the white $\mathrm{GBF}_{4}$ solid. One gram of SSY (disodium salt, SigmaAldrich) was converted to the neutral disulfonic acid through ion exchange of a $50 \mathrm{~mL}$ aqueous solution on Amberlyst 36 resin (Sigma-Aldrich). To this solution was added $1.2 \mathrm{~g}$ of $\mathrm{GBF}_{4}$. Water was then removed by rotary evaporation. The remaining red solid was washed thoroughly with acetone to give about 0.9 g pure $\mathrm{G}_{2} \mathrm{SSY}$ as determined by ${ }^{1} \mathrm{H}-\mathrm{NMR}$ spectroscopy.

Twenty $\mathrm{mg} \mathrm{G}_{2} \mathrm{SSY}$ was dissolved in $0.4 \mathrm{~mL}$ water in an open $4 \mathrm{~mL}$ vial inside a closed $20 \mathrm{~mL}$ vial containing $5 \mathrm{~mL}$ THF. THF vapor diffused into the aqueous solution at room temperature over a period of one week after which elongated red crystals were deposited. ${ }^{1} \mathrm{H}-\mathrm{NMR}$ spectroscopy indicated that the crystals contained two equivalents of $\mathrm{H}_{2} \mathrm{O}$ and one equivalent of THF. Their composition was $\mathrm{G}_{2} \mathrm{SSY}$ THF $2 \mathrm{H}_{2} \mathrm{O}$. The crystals were plank-like with principal faces $\{100\},\{010\}$, and $\{001\}$ (see Supporting Information). The long axis was $<100>$ and the largest faces were $\{100\}$. The crystals were strongly dichroic and most absorbing when the incident light was plane-polarized parallel to $<010>$, perpendicular to the needle axis.

\section{X-ray Crystallography.}

Single Crystal. A G $\mathrm{G}_{2} \mathrm{SSY} \cdot \mathrm{THF} \cdot 2 \mathrm{H}_{2} \mathrm{O}$ crystal described above, measuring $0.29 \times 0.34 \times 0.53 \mathrm{~mm}^{3}$, was mounted on a microloop. Crystals of $\mathrm{G}_{2} \mathrm{SSY}$ THF $2 \mathrm{H}_{2} \mathrm{O}$ turned opaque upon standing in air at room temperature, presumably due to solvent efflorescence. The lattice parameters of another crystal contracted slightly on cooling from $295 \mathrm{~K}\left(V=3105(1) \AA^{3}\right)$ to $100 \mathrm{~K}\left(V=2978.0(9) \AA^{3}\right)$, but no phase changes were observed (See supporting information for thermo-gravimetric analysis). The geometry and intensity data 
were collected with a Bruker SMART APEXII CCD area detector on a D8 goniometer at $100 \mathrm{~K}$. The temperature during the data collection was controlled with an Oxford Cryosystems Series 700+ instrument. Preliminary lattice parameters and orientation matrices were obtained from three sets of frames. Data were collected using graphite-monochromated and $0.5 \mathrm{~mm}$-MonoCap-collimated $\mathrm{MoK \alpha}$ radiation $(\lambda=0.71073 \AA)$ with the $\omega$ scan method. Data were processed using the APEX2 software ${ }^{51}$ for cell refinement and SAINT for frame data reduction. The final unit cell parameters were $a=6.8426(5) \AA$, $b=20.048(1) \AA, c=21.466(2) \AA, V=2944.7(4) \AA^{3}$ for an orthorhombic- $P$ lattice. Multi-scan absorption corrections were applied by using SADABS. The structure was solved using SHELXT, which confirmed the space group Pnna, no. 52 and the structure was refined with SHELXL on $F^{2} .{ }^{52}$ Non-hydrogen atoms were refined with anisotropic displacement parameters. Hydrogen atoms in SSY and G were placed in idealized positions $(\mathrm{C}-\mathrm{H}=0.95 \AA, \mathrm{N}-\mathrm{H}=0.88 \AA$, and $\mathrm{O}-\mathrm{H}=0.84 \AA)$ and included as riding with $U_{\text {iso }}(\mathrm{H})$ $=1.5 U_{\mathrm{eq}}($ non- $\mathrm{H})$. Hydrogen atoms on water molecules were found from the differential electron density maps and refined with a restrained geometry $(\mathrm{O}-\mathrm{H}=0.84 \AA$ and $\mathrm{H} . . . \mathrm{H}$ distance $=1.40 \AA)$. Except for the $\mathrm{SO}_{3}^{-}$groups, one water molecule, and one $\mathrm{G}^{+}$, other atoms in the structure are disordered. Distance restraints were applied to the disordered part in the SSY anion to fit the idealized geometry of the azo tautomer of SSY. The distance restraints were also applied to the disordered $\mathrm{G}^{+}$. The THF solvent molecule could not be modeled sufficiently using the residual peaks from the differential electron density maps, therefore its contribution was subtracted from the diffraction data by the SQUEEZE command in PLATON. ${ }^{53}$ SQUEEZE estimated that each void has a volume of $128 \AA^{3}$ and can hold approximate 42 electrons, which corresponds to a THF $\left(\mathrm{C}_{4} \mathrm{H}_{8} \mathrm{O}, 40\right.$ electrons in total). The data is consistent with the formula derived from NMR.

Powder X-Ray Diffraction. Powder X-ray diffraction (PXRD) was performed with a Bruker D8 Discover Microdiffractometer with the General Area Detector Diffraction System (GADDS) equipped with a VÅNTEC-2000 2D detector. The X-ray beam was monochromated with a graphite crystal $(\lambda \mathrm{Cu}-$ $\mathrm{K} \alpha=1.54178 \AA$ ) and collimated with a $0.5 \mathrm{~mm}$ capillary (MONOCAP). The powder of $\mathrm{G}_{2} \mathrm{SSY}$ THF $2 \mathrm{H}_{2} \mathrm{O}$ was loaded in a $0.8 \mathrm{~mm}$ Kapton capillary and mounted in a horizontal configuration on a sample stage affixed to a five-circle Eulerian cradle. The two-dimensional (2D) diffraction data collection was controlled by the GADDS software. ${ }^{54}$ Two scans with the rotation of the capillary were acquired at the incident angle $\left(\theta_{1}\right)=$ detector angle $\left(\theta_{2}\right)=11^{\circ}$ and $26^{\circ}$, respectively. At each angle, the exposure time was 10 minutes. The sample-to-detector distance was $150 \mathrm{~mm}$. One-dimensional diffraction patterns were generated by integrating the 2D XRD data using XRD2EVAL in the Bruker PILOT software. ${ }^{54}$ The integrated 1D pattern was analyzed by the software DIFFRAC ${ }^{\text {plus }}$ EVA $^{55}$. The simulated 1D powder pattern was calculated by the software Mercury. ${ }^{56}$ From the powder data, there was no evidence of any phases in the bulk sample other than $\mathrm{G}_{2} \mathrm{SSYTHF} 2 \mathrm{H}_{2} \mathrm{O}$. 
Gas Phase Density Functional Theory Calculations. Density functional theory (DFT) calculations were performed with the SIESTA code ${ }^{57}$ using the non-local van der Waals method vdW$\mathrm{DF} 2^{58}$ to describe exchange-correlation. The effective potentials due to the nucleus and core electrons were described using Troullier and Martins ${ }^{59}$ norm-conserving pseudopotentials. Electronic wave functions were expanded in a basis set of atom-centered numerical atomic orbitals. Geometries were optimized using a double-zeta plus polarization (DZP) basis set, while final energies were calculated using an optimized triple-zeta plus polarization (TZP) basis set, an approach that has been shown to accurately model geometries and energies. Hartree and exchange correlation energies were evaluated on a uniform real-space grid of points with a kinetic energy cutoff of 300 Ry. The geometries of the SSY monomer and dimer configurations considered here were fully optimized in a 3D periodic unit cell, with a vacuum region of approximately $20 \AA$ to prevent interaction between periodic images. The binding energy $E_{\text {binding }}$ of each dimer was calculated as $E_{\text {dimer }}-2 \times E_{\text {molecule, }}$, where $E_{\text {dimer }}$ is the energy of each optimized dimer configuration, and $E_{\text {molecule }}$ is the energy of an isolated SSY molecule. To account for basis set superposition error (BSSE), the binding energies were corrected using the Counterpoise (CP) correction method. ${ }^{60,61}$

Implicit Solvent Gas Phase Molecular Mechanics Calculations. An estimate of the solvation free energies of monomers and dimers was calculated using the COSMIC methodology, ${ }^{62}$ an extension of the COSMO model, ${ }^{63}$ implemented in the GULP code. The default GULP van der Waals radii were used. Several additional parameters were also required, the dielectric constant of water, 78.4, the radius of water used to generate the solvent accessible surface (SAS) (1.4 $\AA$ ), and the radius shift of water $(1.3 \AA)$. The number of points and segments per atom that control the fineness of the SAS, were both set equal to 194. A smoothing range of $0.2 \AA$ was used to ensure continuous behavior of the energy surface.

Gas Phase Molecular Mechanics Calculations. The GULP code ${ }^{64}$ was used to optimize the gas phase monomer and dimers produced from the DFT calculations so as to calculate their phonons. The GAFF force field ${ }^{65}$ was used to model the SSY dianion with RESP-A1A partial atomic charges calculated using the RESP ESP charge Derive (RED) Server web service. ${ }^{66}$ The sodium cation potentials were taken from Aqvist. ${ }^{67}$ The interatomic potentials were tapered to zero using a Mei-Davenport-Fernando ${ }^{68}$ function between 8 and $10 \AA$.

Molecular Dynamics Simulations. MD simulations used the same potentials for SSY and $\mathrm{Na}^{+}$as the gas phase molecular mechanics calculations. Water was treated with the SPC/Fw model ${ }^{69}$ optimized to reproduce the thermodynamic, structural, and kinetic properties of liquid water. MD simulations were performed using the LAMMPS code. ${ }^{70}$ Unless otherwise specified, all MD simulations were performed in the NVT ensemble at $300 \mathrm{~K}$ with a 1 fs time step. The temperature was controlled by a length 5 Nosé- 
Hoover chain of thermostats with a relaxation time of 1 ps. The same cut off distances and tapering functions were used as for the GULP calculations, requiring an in-house modification of LAMMPS. The long-range electrostatic interactions were calculated with the PPPM algorithm and an accuracy of $10^{-6}$. The partial atomic charges used and all force field parameters in LAMMPS format are given in the Supporting Information.

Free Energy Calculations. The PLUMED2 plugin $^{71}$ was used to calculate the relative free energy of the stereoisomers of the SSY dimer in water with the metadynamics technique, ${ }^{50}$ here augmented with the well-tempered ${ }^{72}$ and multiple-walkers approaches. ${ }^{73}$ In order to achieve a convergence of the free energy hyper-surface and an optimal exploitation of our supercomputer facilities, we used a starting Gaussian height of $k_{B} T$, a bias factor of 5 for the well-tempered algorithm and 30 independent "walkers", which ran for a combined time of $400 \mathrm{~ns}$.

\section{- RESULTS AND DISCUSSION}

Chromonic Liquid Crystals. LCLCs are lyotropic liquid crystals made from non-covalent associations of small molecules. Small molecules are atypical lyotropic mesogens as they lack micellar structure or covalent, polymeric chains. SSY is among the most well studied LCLC mesogen. Evidence of the LCLC phases of SSY were apparently first reported in PhD theses. ${ }^{74,75,76,77}$ A subsequent thesis of Horowitz in $2005^{78}$ was followed by a full paper ${ }^{17}$ that provoked numerous studies in the past decade aimed at determining the structure of SSY mesophases. ${ }^{18-26}$ In water, SSY exhibits a nematic lyotropic LCLC phase above $\sim 30 \mathrm{wt} \%$ and a hexagonal, columnar phase above $\sim 40 \mathrm{wt} \%$. Like many aromatic LCLCs, SSY mesophases have an X-ray scattering peak at $3.4 \AA$ indicative of close stacking of planar $\pi$ systems in the aggregates. ${ }^{79,95} \mathrm{XRD}$ also indicates that the stacks are one molecule wide with a cross section area of $\sim 1.2 \mathrm{~nm}^{2}{ }^{78}$ Despite a considerable effort at structure determination by small angle XRD, multinuclear NMR spectroscopy, magnetochemistry, rheology, dynamic light scattering, optical absorption spectroscopy, neutron scattering, and polarized light microscopy, no complete picture has emerged from experiment. ${ }^{17-25}$

Solid State Structure. Sulfonated dyes tend to be difficult to crystallize. ${ }^{80,81}$ This judgment is supported by our experience as well as anecdotal evidence. ${ }^{82,83}$ On the other hand, the Cambridge Structural Database has been enriched with sulfonated organic compounds in recent years, following the discovery that sulfonated aromatic compounds are very well organized by guanidinium counterions. ${ }^{84,85,86,87,88}$ Our attempts to crystallize SSY with a variety of simple cations $\left(\mathrm{Na}^{+}, \mathrm{K}^{+}, \mathrm{NH}_{4}^{+}\right.$, $\mathrm{H}_{3} \mathrm{~N}^{+}-\mathrm{CH}_{2} \mathrm{CH}_{2}-\mathrm{NH}_{3}{ }^{+}$, as well as protonated glycine and $L$-alanine) produced very fine anisotropic red needles too small for X-ray analysis. We therefore sought to crystallize SSY in a so-called guanidiniumsulfonate (GS) framework, a strategy previously employed with sulfonated azobenzenes. ${ }^{89,90}$ 
Despite the fact that the C-S bonds in SSY are not co-linear, bent disulfonates have crystallized with $\mathrm{G}^{+}$ ions to form puckered lamellar GS frameworks. ${ }^{91,92,93,94}$ Distorted GS hydrogen bond sheets can pucker thereby serving to accommodate even disulfonates without parallel C-S bonds. These data suggested that SSY, a banana shaped disulfonate with non-colinear C-S bonds, can be also crystallized with $\mathrm{G}^{+}$ions.

X-ray analysis revealed that $\mathrm{SSY}^{2-}$ and $\mathrm{G}^{+}$do not crystalize as a classical GS framework, but rather as colinear, disordered, one-dimensional stacks of SSY molecules where $\mathrm{G}^{+}$ions fill the voids, in addition to two equivalents of water and one of THF. The SSY molecules are planar in the crystal structure (excepting the sulfonate oxygen atoms). The molecules are disordered about the diad axes parallel to $c$ $(1 / 4,0, z)$, as illustrated in Figure 1a. These disordered virtual pairs are adjacent to a second disordered virtual pair - virtual because the two sites related by the diad axes cannot both be occupied simultaneously - directly above and below by $\pm a / 2$ or $\sim 3.4 \AA$. The pairs are related to one another by a glide reflection as shown in Figure 1b. The stacking along the $a$ axis is responsible for the elongation of the crystals in this direction. Given a disordered pair and another pair immediately above or below (Figure 1b), two distinct geometries for adjacent SSY molecules in the crystal in the absence of disorder can be gleaned, an enantiomorphous pair of dimers of symmetry $C_{2}$ and a centrosymmetric dimer $C_{i}$, as depicted in Figure 2. Figure 3 illustrates explicitly how the positions in adjacent unit cells, constrained by the necessity of placing anionic moieties on opposite sides of a stack, produce an average cell with four half-molecules related to one another by a site of approximate $C_{2 h}$ symmetry (though crystallographically only a site of $C_{i}$ symmetry). Even though the disorder muddles the distinction between the hydrazone and azo tautomers (Chart 1), NMR spectroscopy ${ }^{95}$ and Raman scattering ${ }^{96}$ clearly support the hydrazone tautomer and the analyses here were performed under this assumption. The two symmetry independent $\mathrm{G}^{+}$ ions in the $\mathrm{G}_{2} \mathrm{SSY} \cdot \mathrm{THF} \cdot 2 \mathrm{H}_{2} \mathrm{O}$ structure connect neighboring SSY disordered dimers through a series of one-dimensional hydrogen bonded ribbons (along the $a$ axis).

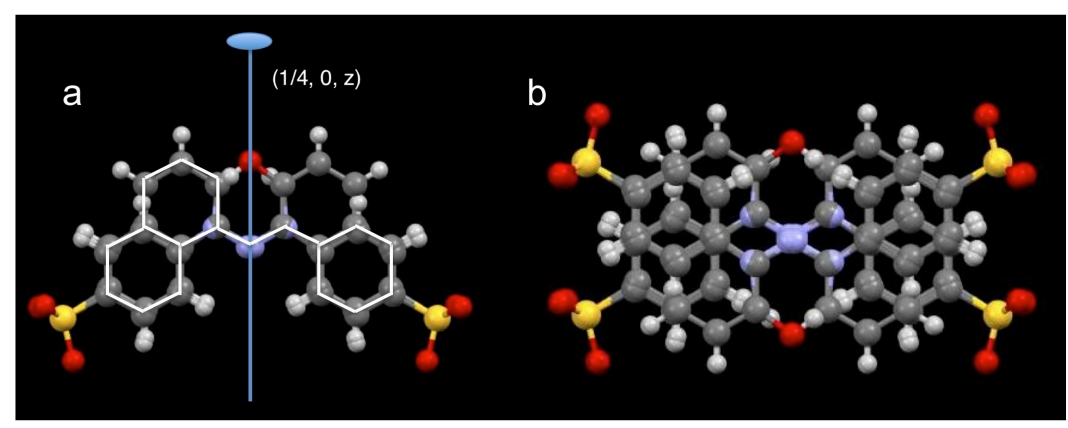

Figure 1. (a) A pair of sunset yellow molecules disordered about a diad axis. Each has crystallographic occupancies of 0.5.. The skeleton of one of the molecules is superimposed in white. (b) Two adjacent pairs of disordered sunset yellow molecules in parallel planes separated by $a / 2$. 

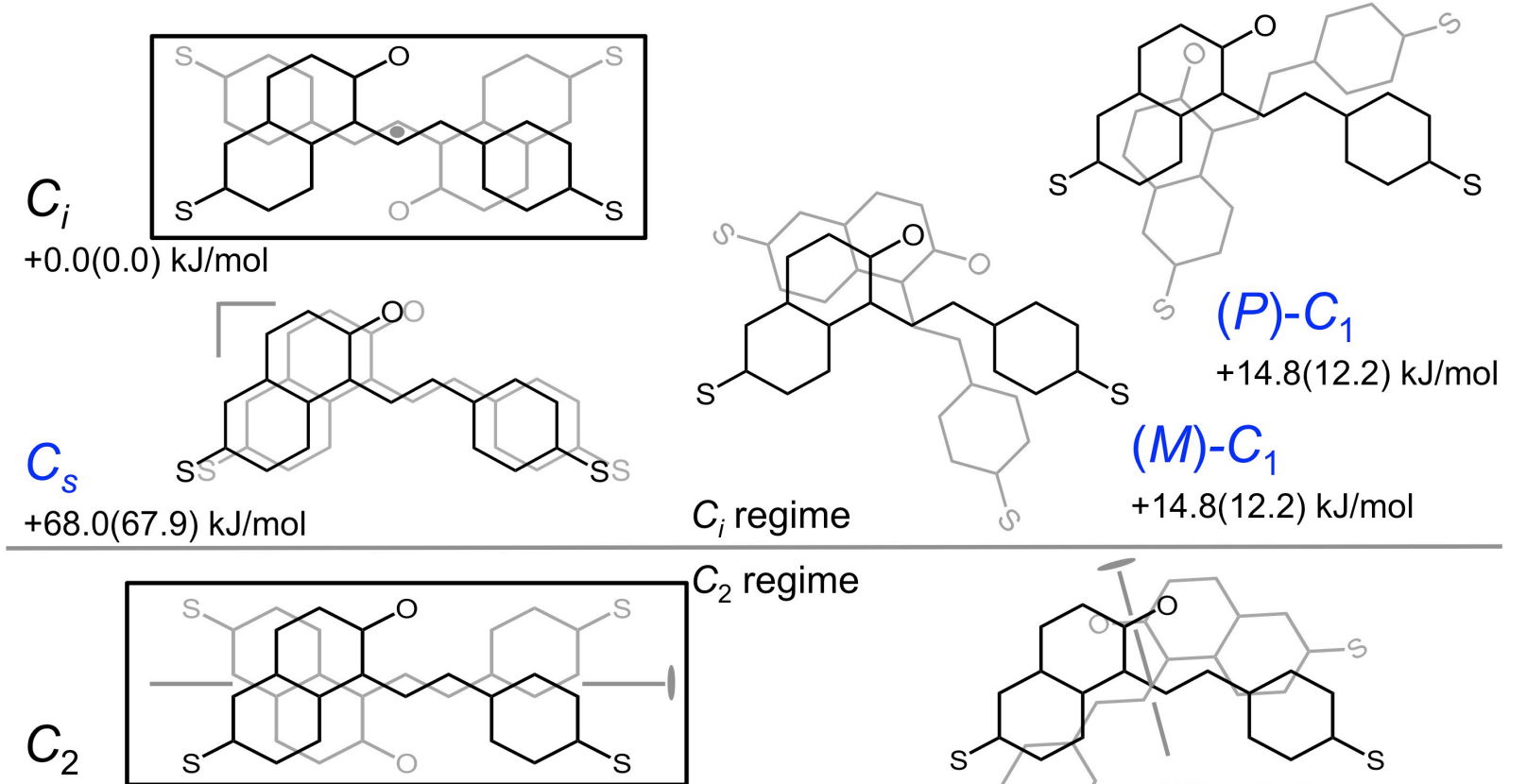

$0.1(0.1) \mathrm{kJ} / \mathrm{mol}$
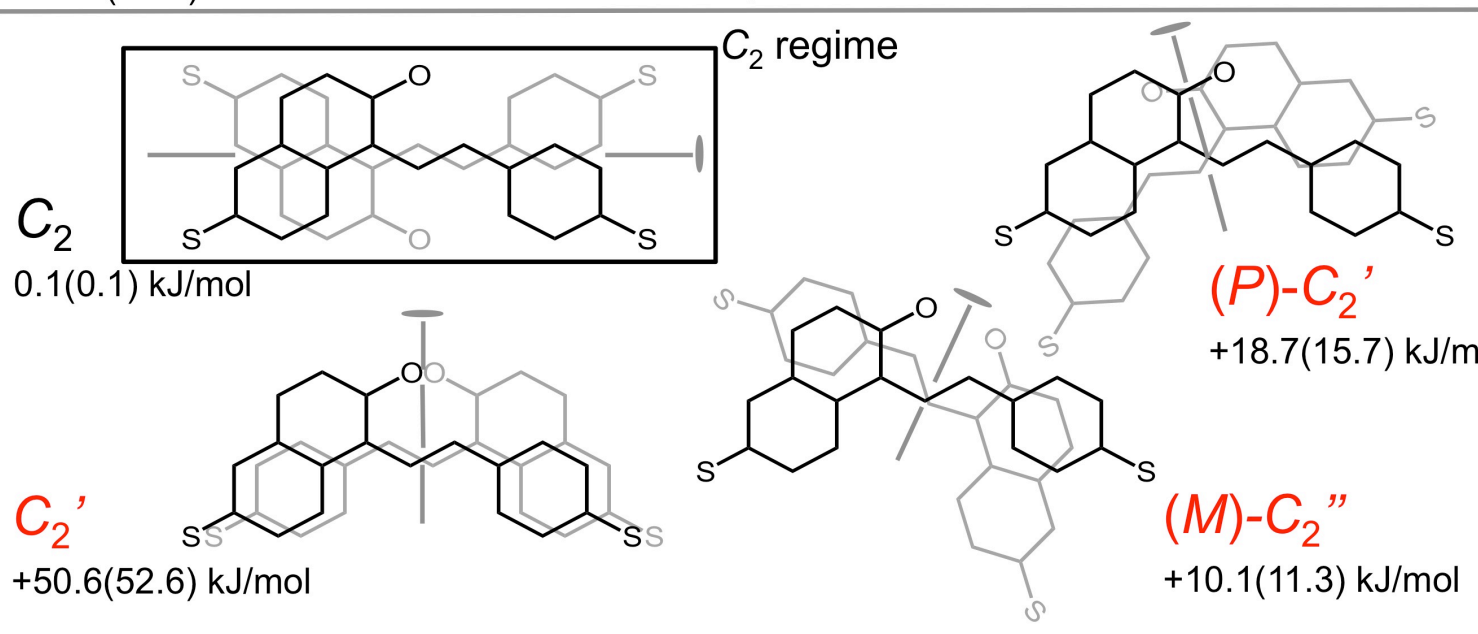

$(P)-C_{2}$

$+18.7(15.7) \mathrm{kJ} / \mathrm{mol}$

$+50.6(52.6) \mathrm{kJ} / \mathrm{mol}$

Figure 2. Schematic sunset yellow dimers and relative gas-phase quantum chemical (force field) energies in $\mathrm{kJ} / \mathrm{mol}$ relative to the centric structure, $C_{i}$, in the upper left corner. Gray molecules are below black molecules. Only the sulfur atoms and the carbonyl oxygen atom are denoted explicitly in these representations. The symmetry descriptors refer to the relationships between molecules. Equi-energetic $C_{i}$ and $C_{2}$ structures are observed in the disordered crystal structure. The are enclosed in black boxes.

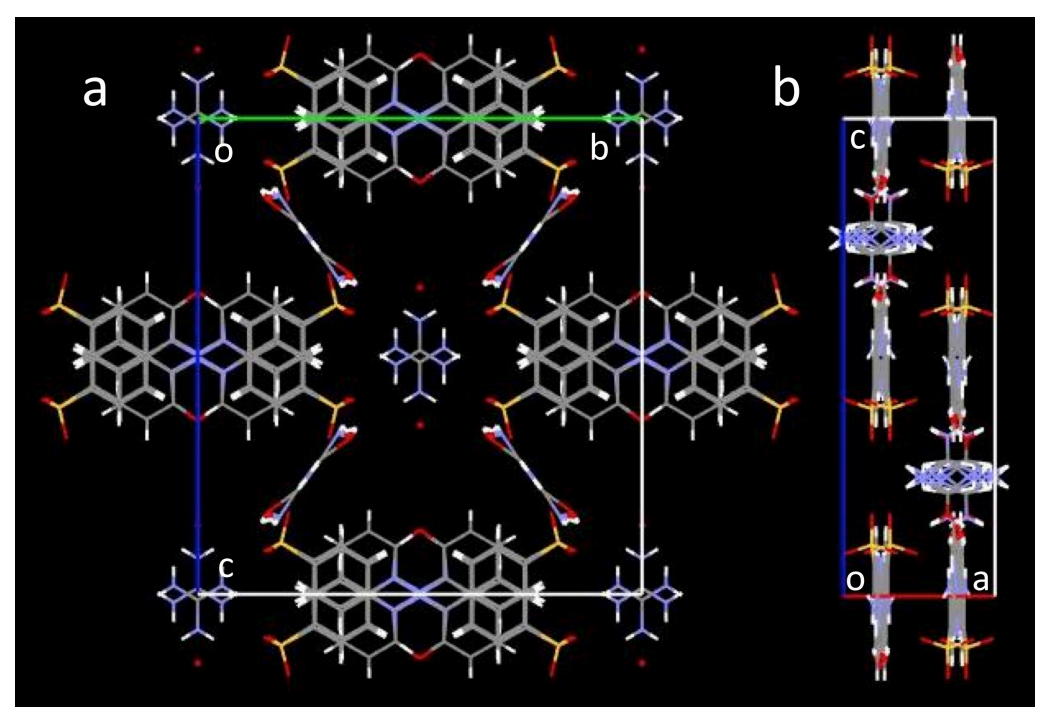

Figure 3. Crystal packing of $\mathrm{G}_{2} \mathrm{SSY}^{\cdot} \mathrm{THF}^{\cdot} 2 \mathrm{H}_{2} \mathrm{O}$. (a) Viewed along the $a$ axis, and (b) along the $b$ axis. 


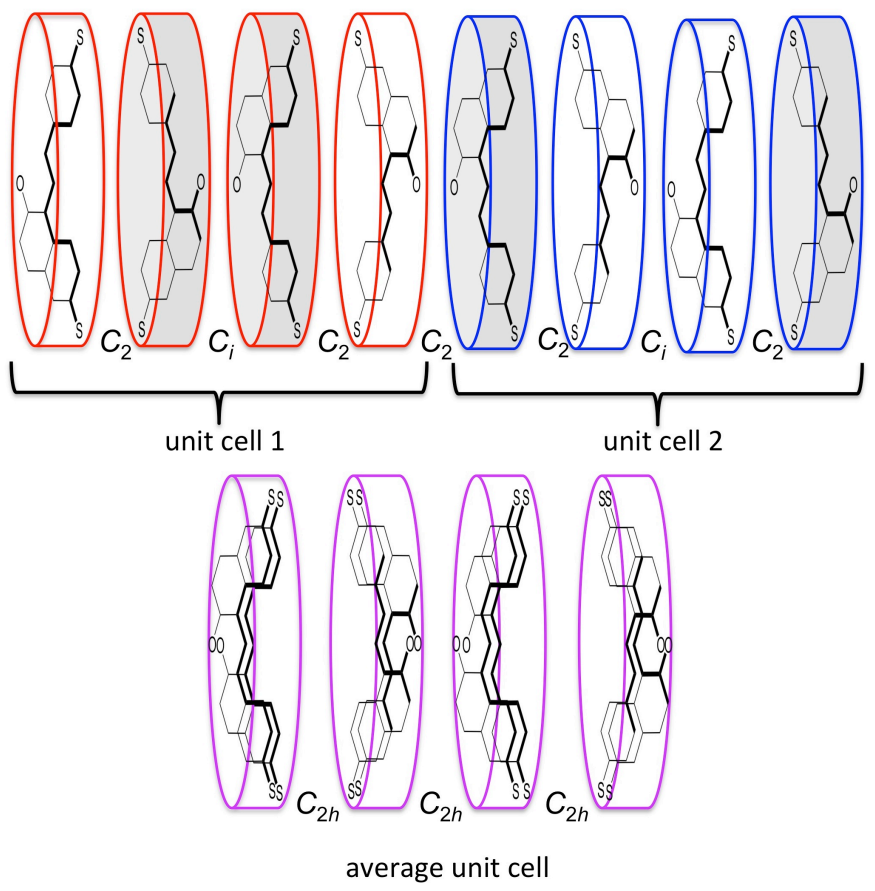

Figure 4. Averaging of the contents of adjacent red and blue unit cells (top) in which molecules related by approximate diad axes and centers of symmetry conspire to place tetrads of molecules at half occupancy surrounding an approximate special position of $C_{2 h}$ symmetry, now purple (bottom). With respect to the first molecule at left, shaded disks denote SSY molecules with their enantiotopic faces exposed on the left side.

Stereochemistry. The pairwise associations observed in the crystal structure of $\mathrm{G}_{2} \mathrm{SSY} \cdot \mathrm{THF} \cdot 2 \mathrm{H}_{2} \mathrm{O}$, have symmetries $C_{2}$ and $C_{i}$ as illustrated in Figures 2 and 4. Based on the observations of Chami and Wilson ${ }^{49}$ that stacks of SSY molecules in solution are dynamic, we envisioned a series of possible stationary points on the potential energy hypersurface of a dimer of SSY. These are reproduced in Figure 2. Optimized gas phase structures were initially computed using DFT using the vdW-DF2 functional, where the $\mathrm{Na}^{+}$ counterions were positioned within the sulfonate oxygen triads along the C-S axes. The quantum mechanical calculations predicted that $C_{i}$ is more stable than $C_{2}$, by just $0.1 \mathrm{~kJ} / \mathrm{mol}$; the two crystallographic dimers have nearly equal energy. They are enclosed in black boxes in Figure 2. Both crystallographic dimers position their sulfonates on opposite sides while maximizing dispersion forces. The disordered crystal structure represents a superposition of $C_{2}$ and $C_{i}$ pairs (Figure 4). $C_{2}$ and $C_{2}{ }^{\prime}$ indicate orthogonal diad axes along the long and short axes of the molecules respectively. $C_{s}$ indicates a pair of molecules related by translation along the axis perpendicular to the mean molecular planes. As the molecules are themselves flat, barring sulfonate oxygens, this pair is related by mirror symmetry. Structures with perfectly eclipsing sulfonates $C_{2}{ }^{\prime}$ and $C_{s}$ are much higher in energy by 50.6 and 68.0 $\mathrm{kJ} / \mathrm{mol}$, respectively. These structures can distort by skewing the top structure clockwise or counterclockwise with respect to the bottom structure (in gray). $(P)-C_{2}{ }^{\prime}$ and $(M)-C_{2}{ }^{\prime \prime}$ are diastereomers derived from $C_{2}$ ' by clockwise or plus $(P)$ and counterclockwise or minus $(M)$ rotations of the top structure with respect to the one below it (set colored red). Note: Ordinarily, a pair of isomers $(P)$ and $(M)$ 
might indicate a pair of enantiomers. Here, the 'plus' and 'minus' indicates deviations from the fully eclipsed structure $C_{2}{ }^{\prime}$. Diastereomeric $(M)-C_{2}{ }^{\prime \prime}$ and $(P)-C_{2}$ ' configurations have relative energies of 10.1 and $18.7 \mathrm{~kJ} / \mathrm{mol}$, respectively. $(P)-C_{1}$ and $(M)-C_{1}(14.8 \mathrm{~kJ} / \mathrm{mol})$ are indeed enantiomers derived from $C_{s}$ by clockwise and counter clockwise rotations, respectively. The energies from the interatomic potential calculations are also included in Figure 2 parenthetically. The structures above the median line in Figure 2 can interconvert with one another by rotations around the stacking axis, as can the structures below the median line. Structures in either half of the figure can only interconvert by flipping over, an energetically prohibitive process.

We were unable to define the nature of the stationary points using DFT due to the high computational cost of evaluating the second derivatives for calculating the phonon spectra of the dimers. Such calculations with interatomic potentials are computationally cheap, however, especially when the computational engine used has analytical expressions for the second derivatives, as in GULP. The quality of the interatomic potentials can be assessed by comparing the energy differences and geometries calculated by the force field with the results of DFT. The agreement between the two methods is exceptional with a maximum deviation in the calculated energy differences of only $3 \mathrm{~kJ} / \mathrm{mol}$. The structures of each stationary point calculated using the two methods again agree well (In each case, they are near-perfectly superimposed as shown in the Supporting Information.) and the interatomic potentials employed are reliable from this point of view. The GULP calculations show that the $C_{s}$ and $C_{2}$ ' configurations are not minima as they have at least one large negative phonon. The remaining stationary points are all minima, although the $(M, P)-C_{1},(M)-C_{2}$ " and $(P)-C_{2}$ ' are shallow. The optimized configurations of all three had a negative phonon at $\sim-5 \mathrm{~cm}^{-1}$, which could only be removed by restraining the $\mathrm{Na}^{+}$counterions to positions within the sulfonate oxygen triads along the C-S axes.

The gas phase binding energy of the dimers is given by $E_{\text {binding }}=E_{\text {dimer }}-2 \times E_{\text {molecule }}$ and for the most stable dimer is calculated to be $-82.6 \mathrm{~kJ} / \mathrm{mol}$ by DFT and $-88.2 \mathrm{~kJ} / \mathrm{mol}$ using potentials.

It is a simple matter to build a polymer structure from a stack of pairwise $C_{i}$ and $C_{2}$ relationships. In a random distribution of pairwise interactions, we can presume that there will exist lengths related by only the center of symmetry, lengths related by only the long axis diad, and lengths in which there is a stochastic arrangement of dimers with these two pairwise relationships. Borrowing the language of conventional polymer stereochemistry, ${ }^{97}$ we will call these syndiotactic, isotactic, and atactic. In syndiotactic stretches the stereogenic elements - the pairwise orientations of the SSY molecules in a stack - are related by a symmetry operation of the second kind. In isotactic stretches, the stereogenic elements are related by a proper rotation, a symmetry operation of the first kind. There is no persistence in atactic lengths. Correlations in the crystal structure could be assessed through an analysis of the diffuse scattering, an analysis we will aspire to make in future. 
Stereodynamics. Stereodynamics of SSY aggregates were first investigated by Chami and Wilson. ${ }^{49}$ They described reorientations within pre-chromonic stacks by rotations of $180^{\circ}$ around the stacking axis and monitored conformational changes in their simulations by plotting the dot product of $\mathrm{C}=\mathrm{O}$ vectors in parallel planes in a centric $C_{i}$ stack (a syndiotactic block as pictured in Figure 5). The cosine of this angle was described as oscillating between \pm 1 , however. Inspection of the published traces indicates that the cosine actually varied between -1 and +0.7 , with a small number of very brief excursions to +1 . The mean position of the molecules is characterized by $\cos (\sim 0.7)$ or $\sim 45^{\circ}$. The molecules do rotate about the stacking axis but not all the way around. Rotations of one molecule by $180^{\circ}$ in a $C_{2}$ or $C_{i}$ pair will produce the transition state geometries with eclipsing sulfonate groups $C_{2}{ }^{\prime}$ and $C_{\mathrm{s}}$ respectively, as depicted in Figure 2. Eclipsing of like-charge groups is unlikely on electrostatic grounds, and consequently it is poorly populated in the Chami and Wilson MD simulations. Quantum mechanical simulations support this observation, with $C_{2}{ }^{\prime}$ and $C_{\mathrm{s}}$ configurations having relative energies of 50.6 and $68.0 \mathrm{~kJ} / \mathrm{mol}$,

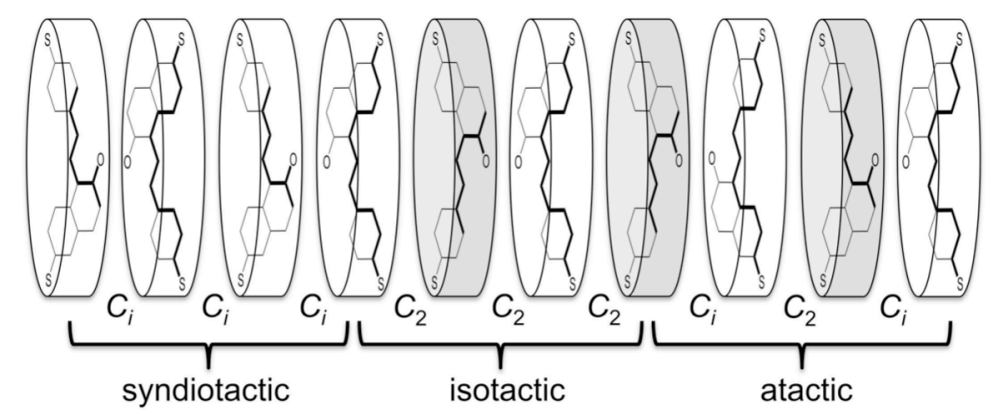

Figure 5. Stack of ten SY molecules with pairwise relationships derived from the crystal structure. With respect to the first molecule at left, shaded disks have their molecule's enantiotopic face exposed on the left side. Diad axes exchange enantiotopic faces. Centers of symmetry do not.

respectively, compared with the non-eclipsed dimer configurations, which have energies ranging from 0$18.0 \mathrm{~kJ} / \mathrm{mol}$. Simulations of a centric $C_{i}$ stack provide no information about the $C_{2}$ regime (Figure 2) of SSY molecules as a $C_{i}$ dimer cannot be transformed to a $C_{2}$ dimer via rotation about the stacking axis. This can only occur by the prohibitively energetic process of exchanging faces of SSY molecules, that is, flipping. Consequently, we performed long timescale (100 ns) MD simulations on a dimer in a $50 \AA^{3}$ box of water, starting from both a $C_{i}$ and $C_{2}$ dimer configuration.

In Figure 6, a plot of the dot product of the $\mathrm{C}=\mathrm{O}$ vector on each molecule is presented for the two simulations corresponding to $C_{\mathrm{i}}$ pairs (Figure 6a) and $C_{2}$ pairs (Figure 6b). Figure 6a shows that starting from the $C_{i}$ dimer, the carbonyl varies from a strictly antiparallel $C_{i}$ configuration $\left(180^{\circ}\right)$ to something falling far short of fully eclipsed with a cosine of $\sim 0.6$. This value is smaller than the 0.7 observed by Chami and Wilson ${ }^{49}$ because we are analyzing isolated dimers rather than the central dimer in a stack of four molecules, and because of differences in water and point charge models (Chami and Wilson did not indicate which charge model they used). The transitions for the $C_{2}$ dimer are even more interesting. The 
dimers stays in the $C_{2}$ configuration for $\sim 52 \mathrm{~ns}$, then jumps to the $(P)-C_{2}$ ' structure with antiparallel $\mathrm{C}=\mathrm{O}$ vectors can shown in Figure 2. This dimer is short-lived; it transforms back to $C_{2}$ after $8 \mathrm{~ns}$, where it remains for $16 \mathrm{~ns}$. Then it converts to $(P)-C_{2}{ }^{\prime}$ again but this time for only $4 \mathrm{~ns}$. It transitions back to $C_{2}$ at $80 \mathrm{~ns}$ and then to $(M)-C_{2}$ " at $90 \mathrm{~ns}$ and remains in this configuration until the end of the simulation at 100 ns. It is noteworthy that the angle between $\mathrm{C}=\mathrm{O}$ vectors is a good discriminator between $(M)-C_{2}$ " and $(P)$ $C_{2}$ ' dimers but it does not discriminate well between $C_{2}$ and $(M)-C_{2}$ " (although careful inspection of Figure $6 \mathrm{~b}$ reveals that the former oscillates about $\sim 0.25$, whereas the latter oscillates about $\sim 0.5$, although the oscillations have a magnitude in excess of 0.5 ).
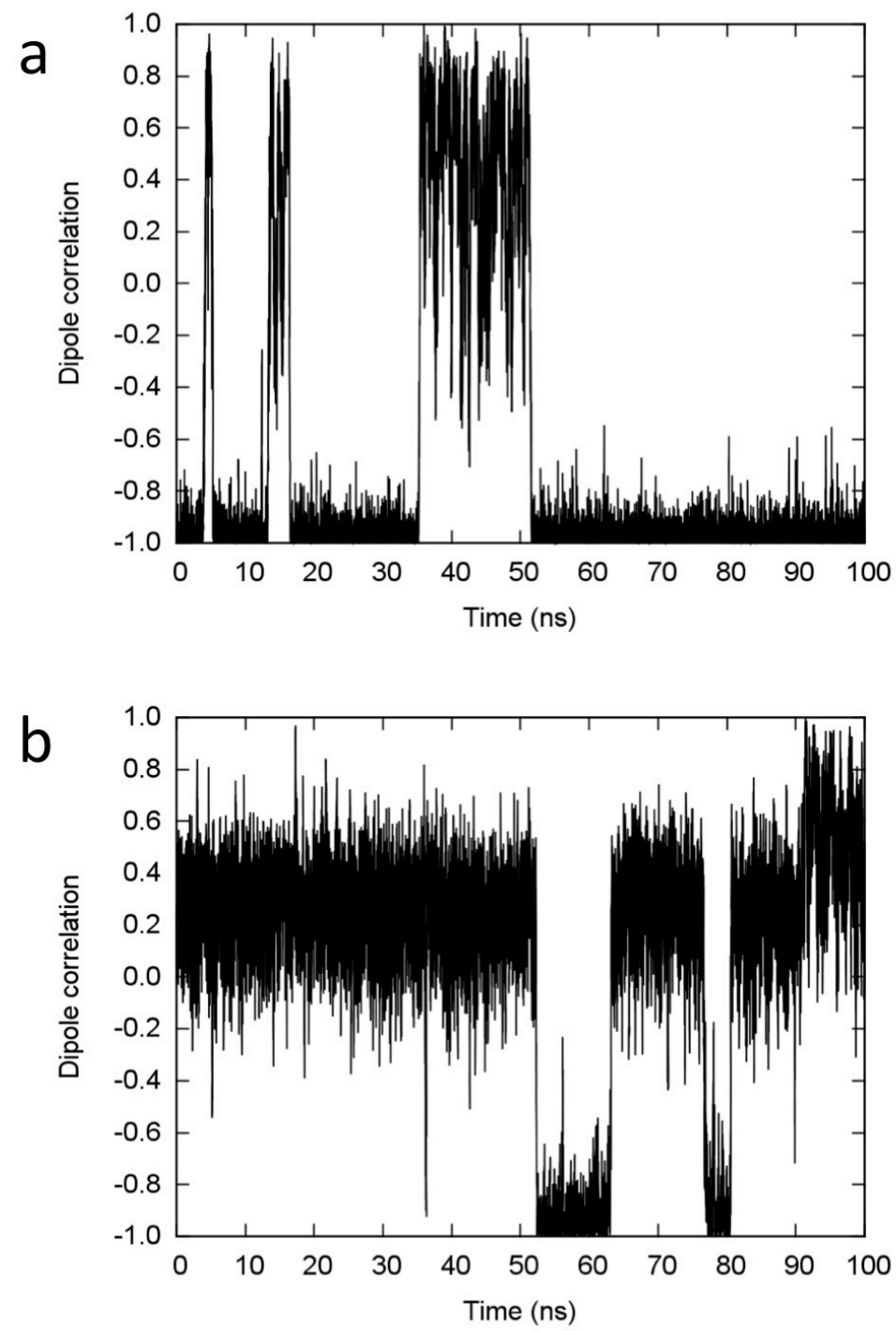

Figure 6. (a) Dot product of the carbonyl unit vectors for the structure in the $C_{i}$ regime, and (b) for the $C_{2}$ regime. 
Despite running long simulations (100 ns each), we did not observe a large enough number of transitions to be able to calculate the relative free energy of the stereoisomers via a simple population analysis. We therefore turned to metadynamics to better characterize the relative stability. Here, the sampling of configurational space was enhanced so as to retrieve a free energy hyper-surface that connects all the stereoisomers of SSY. For a full description of the method we refer the reader to the published literature ${ }^{98}$ as only a brief description of its working principles is provided here. Metadynamics is based on two ideas: $(i)$ the dimensional reduction of the configurational space from the $3 N-3$ degrees of freedom to a few collective variables (CVs) that describe the process of interest, and (ii) the addition of a time dependent potential that discourages the system from remaining in the same configuration. The timedependent potential is constructed by periodic additions of Gaussian functions to the sub-space of the potential energy surface defined by the CVs. It can be demonstrated that upon convergence of the simulation the final bias potential is equal to the inverse of the system free energy, projected on the CV's sub-space. An essential prerequisite to a meaningful free energy hyper-surface from a Metadynamics calculation is that the chosen CVs clearly discriminate among the various minima and closely approximate the true reaction coordinate through the various transitions states. We have previously demonstrated the dot product of the $\mathrm{C}=\mathrm{O}$ vectors does not clearly discriminate between all the SSY stereoisomers. More discriminating CVs are requisite. We chose three CVs that effectively discriminate all the stereoisomers and describe their interconversion. They are the two intra-dimer S-S distances corresponding to the phenyl and naphthyl $\mathrm{S}$ atoms on one molecule with that on the other molecule, respectively, and the dot product between two unit vectors normal to the naphthyl rings. Trajectories from the long MD simulations emerged as a testing ground for these new CVs, albeit producing 4D free energy surfaces that were difficult to visualize. Therefore, extensive use of projections of the free energy surface on a subset of these CVs is more practical

The two unique S---S vectors do not uniquely describe configurational space. It is indeed possible to have quite different structures with the same pair of S---S distances, but most of these structures are much higher in energy and are therefore not represented on the metadynamics potential energy surfaces. The third $\mathrm{CV}$ is used to distinguish between the possible stacking arrangements, the dynamic $C_{i}$ and $C_{2}$ spaces. If the SSY molecules are stacked in the $C_{i}$ regime the third CV has a value of +1 , while if they are stacked in the $C_{2}$ regime it assumes a value of -1 . Thermal movement during the MD simulations causes fluctuations to values less than $|1|$. The two main branches of the potential energy surface are designated " $\cos \theta>0 "$ and $" \cos \theta<0$ " for the \pm 1 configurations, respectively.

In Figure 7, we show the free energy maps as a function of the two S-S distances for limiting $C_{i}$ and $C_{2}$ configurations. These were obtained by considering separately the Gaussians for the positive $\cos \theta$ (Figure 7a) and negative $\cos \theta$ (Figure $7 \mathrm{~b}$ ) stacking and by calculating the thermodynamic average along the $\cos \theta$ $\mathrm{CV}$. The positive $\cos \theta$ stacking map recognizes the two free energy minima as $C_{i}$ and $(M, P)-C_{1}$ 
enantiomers. $C_{i}$ conformation represents the global free energy minimum for the system and all the free energies are reported relative to its energy. The $C_{1}$ state, composed of the two enantiomers $(M, P)-C_{1}$ is only marginally higher in free energy $(\sim 5 \mathrm{~kJ} / \mathrm{mol})$ with respect to the $C_{i}$ state and it is likely to be found in solution. The interconversion between the two conformations has a moderate activation energy (5-10 $\mathrm{kJ} / \mathrm{mol}$ ) and was observed multiple times during the unbiased MD simulation. The transformation of one structure does not occur as a simple rotation of one molecule with respect to the other, but there can be a partial translation or detachment of the molecules to facilitate the passage of one sulfate group around the other while minimizing electrostatic repulsion. The enthalpy difference in vacuum is $\sim 2.5$ times higher than the free energy difference in water, indicating that the medium has significantly stabilized the $C_{1}$ state compared to $C_{i}$. Molecular arrangements compatible with the $C_{2}^{\prime}$ structure, although never completely eclipsed, were observed only a couple of times during the metadynamics calculations with the classical force field, which suggests that this is not a free energy minimum, consistent with the gas phase enthalpy calculations using the same force field. 

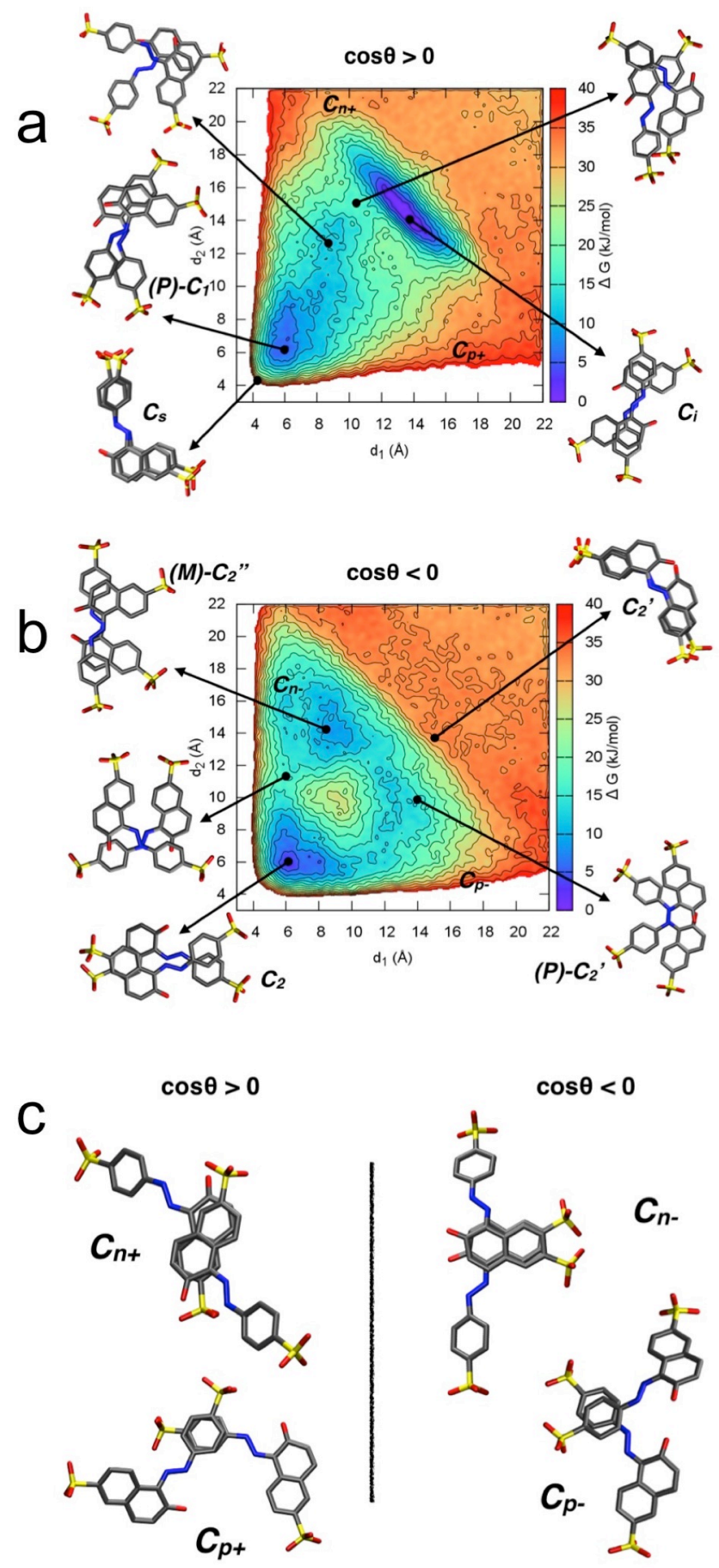

Figure 7. 2D free energy maps obtained from the metadynamics simulations as a function of the two S-S distances for the positive $\cos \theta$ (a) and negative $\cos \theta$ (b) stacking of the molecules in the dimer configurations. Molecular arrangements corresponding to various regions of the free energy maps are also shown. $C_{n^{ \pm}}$and $C_{p^{ \pm}}$are dimer configurations that could lead to stacking faults (c). ${ }^{79}$

In the free energy map for the negative $\cos \theta$ stacking (Figure 7b), two minima correspond to $C_{2}$ and $(M)$ $C_{2}$ " with the latter around $2.5 \mathrm{~kJ} / \mathrm{mol}$ higher (compared to $11.3 \mathrm{~kJ} / \mathrm{mol}$ in the gas phase). Unlike the previous case, the inter-conversion between the two conformers can occur via two channels, a wide 
looping channel which has the $(P)-C_{2}$ ' structure as an intermediate configuration, and a narrow pathway where the transition state does not show any stacking of the aromatic rings. In both cases, the free energy barrier for the inter-conversion between the $C_{2}$ and $(M)-C_{2}$ " conformers is moderate $(\sim 10 \mathrm{~kJ} / \mathrm{mol})$ and can be easily overcome during the unbiased simulations.

Despite the fact the conformers in the $C_{i}$ and $C_{2}$ regime have very similar energies $(\Delta G \sim 2 \mathrm{~kJ} / \mathrm{mol})$, they are separated by a free energy barrier of the order of $28 \mathrm{~kJ} / \mathrm{mol}$, a consequence of the necessity of breaking van der Waals contacts and increasing the aqueous solvation of the aromatic moieties. Interconversion will be a rare event at room temperature. The metadynamics indicate that interconversion occurs through disassociation/reassociation of separated pairs, as opposed to a coordinated SSY flip. Because of the size of our simulation cell $(\sim 50 \AA)$ and of the constraint we imposed on the S-S distances to be shorter than $22 \AA$ we did not completely explore the dimer dissociation process. Assuming that the free energy barrier for the change in stacking is smaller than that for a complete dissociation, the barrier for the rupture of the dimer will be larger than $28 \mathrm{~kJ} / \mathrm{mol}$. The binding free energy of a dimer in water was also calculated using the COSMIC implicit solvation scheme, which predicts a value of $28.5 \mathrm{~kJ} / \mathrm{mol}$, in surprisingly good agreement with the metadynamics result. .

Stereochemistry and Colligative Properties. Chains in LCLCs grow by attractive interactions in water, compensated by a decrease in entropy as the number of aggregates decreases. The average number of molecules $\left\langle n>^{99}\right.$ in a polydisperse distribution aggregates is given by $<n>=\sqrt{\phi} e^{-\alpha / 2}$, where the scission free $(\alpha)$ energy in units of $k_{B} T$ is independent of chain length, and $\phi$ is the volume fraction of the dye.

One of the outstanding puzzles in LCLC science is the failure of Onsager's theory of rigid rods ${ }^{100,101,102}$ to predict the volume fraction of the isotropic-to-nematic transition. Onsager's athermal theory of the transition predicts that $\phi(L / D) \approx 4$, where $\phi$ is the volume fraction of the aggregates, $L$ is the average length of a stack, and $D$ the diameter. Tomasik and Collings reviewed related theoretical and computational contributions subsequent to Onsager. ${ }^{103}$ For SSY, with $L=3.5 \mathrm{~nm}$ and $D=1 \mathrm{~nm}$, parameters from X-ray diffraction, the product $\phi(L / D)$ is only $1.3 .^{79,103,104} \mathrm{In}$ order to account for this discrepancy, it was proposed that the actual length of rods, $L$, may indeed by longer than the correlation length $\xi_{L}$ obtained by experiment. It was proposed that 'connectedness' might persist for much larger distances with shift junctions (stacking faults) or Y-junctions (forks) that lead to a lower apparent length, $\xi_{L} \cdot{ }^{79}$ NMR measurements of diffusion lengths support the notion of aggregates larger than those given by $\xi_{L}{ }^{22}$ An explicit conformational analysis coupled with detailed simulations can in principle refute or support such structural considerations. The metadynamics simulations show that shift junctions corresponding to regions of the free energy surfaces labeled $C_{n}^{ \pm}$(predominantly naphthyl ring overlap) 
and $C_{p^{ \pm}}$(predominantly phenyl ring overlap) are populated. ${ }^{79}$ In particular, the $C_{n^{-}}$configuration, although being higher in energy than the $C_{2}$ structure, sits in a flat free energy basin and can therefore have a lifetime long enough to accommodate the adsorption of other SSY molecules. On the other hand, forks do not occur for two reasons: (1) The bridging molecule provides an $C_{n}$-type overlap to one column and the less stable $C_{p^{-}}$overlap to the other column, and (2) each column is negatively charged so they will be highly repellent in close proximity; only a fraction of the $\mathrm{Na}^{+}$counterions are bound by the SSY molecules at any given time. To evaluate the stability Y-junctions, we placed such a structure with 4 SSY molecules (Figure 9a) in each 'tyne' in a $50 \AA$ cube box of water and ran molecular dynamics for $100 \mathrm{~ns}$. The Y-junction quickly devolved into isolated stacks containing 4 and 5 SSY molecules (Figure 9b) within $1 \mathrm{~ns}$, with the originally shared SSY molecule joining the stack where it had the $C_{n}$-type overlap. The two stacks remained independent for the remaining $99 \mathrm{~ns}$ of the simulation.

The metadynamics calculations, as discussed above, predict a scission energy of about $28 \mathrm{~kJ} / \mathrm{mol}$. The scission energy of $\sim 7 k_{B} T(18-19 \mathrm{~kJ} / \mathrm{mol})$, based on the correlation length established from the full width at half maximum of the $3.4 \AA$ X-ray $\operatorname{peak}^{17,79}$ or by the spectral changes as a function of concentration, ${ }^{3,17,79}$ is smaller than that estimated by metadynamics for the disassociation of a dimer. (The value of $\sim 7 k_{B} T$ is more or less a consensus value for the scission energy at room temperature at volume fractions below which the mesophases form, but other values have been reported too. ${ }^{21}$ ) This is to be expected if the correlation length is controlled by slipped stacks, rather than discrete stacks, as the free energy to slip a stack is significantly less than the energy required to completely separate them. Indeed Figure $7 \mathrm{~b}$ shows that the free energy required to create the $C_{n^{-}}$configuration is $\sim 15 \mathrm{~kJ} / \mathrm{mol}$, which is a much better match to the experimental estimate, providing further evidence that slipped stacks control the correlation length. 
a
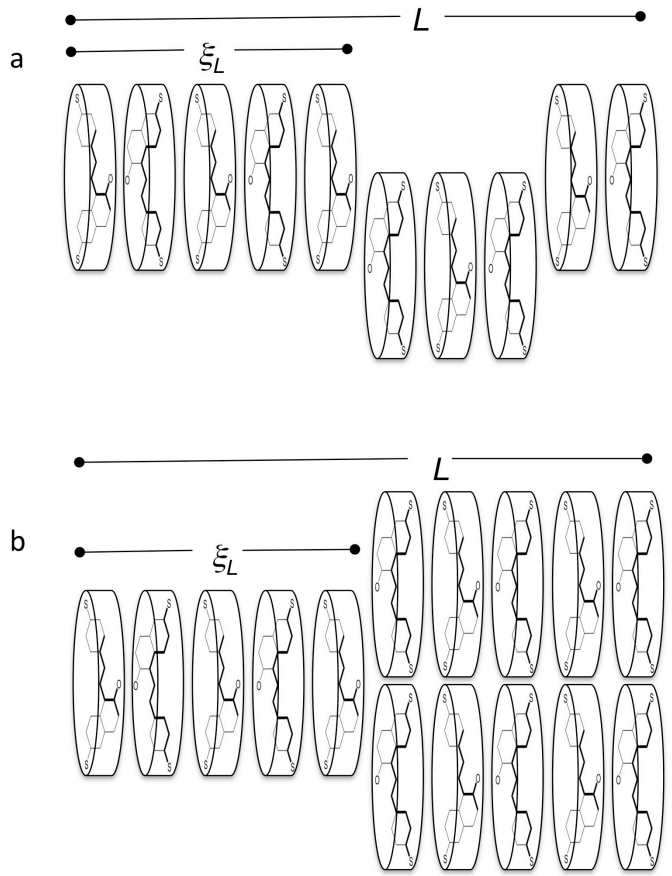

Figure 8. Defective sunset yellow columns, adopted from ref. 79, proposed in order to establish a correspondence with X-ray scattering data and theories for predicting the concentration of the isotropic to nematic transition. (a) Slipped stack. Stacking fault in $C_{i}$ stack which serves to lessen the correlation length $\xi_{L}$ while preserving connectedness in much longer structures of length $L$. (b) Y-junction, likewise in $C_{i}$ stacks.

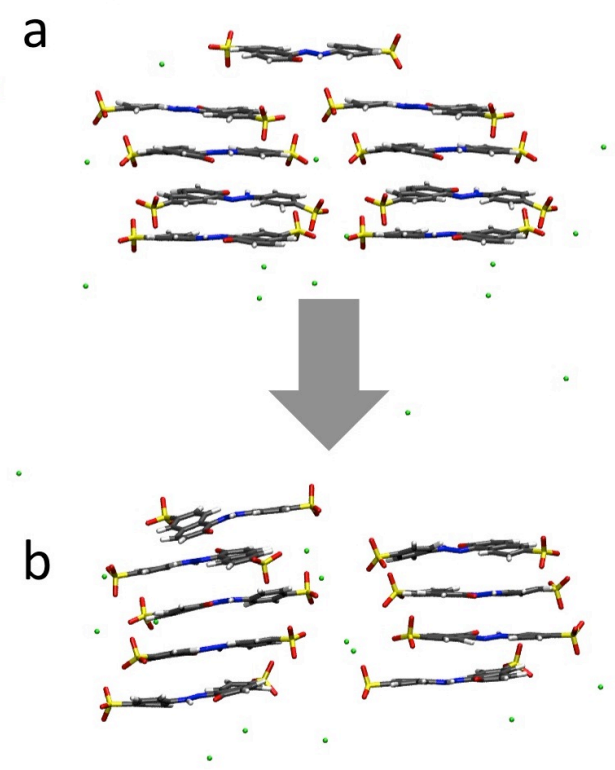

Figure 9. Y-junctions (a) are not stable with respect to molecular dynamics simulations, and quickly devolve into a pair stacks (b). This is because only a fraction of the sodium ions (green dots) are bound, leaving stacks with appreciable negative charge on their perimeters. 


\section{- CONCLUSIONS}

A single crystal structure of a guanidinium salt of SSY, a prototypical LCLC, is reported. The disorder in the crystal structure captures the rich stereochemistry of LCLC stacks built from dissymmetric flat molecules. Quantum chemical, molecular dynamical, and metadynamical computational studies of the associations of pairs of SSY molecules reveals that the dimer configurations observed in the crystalline state also are built into chromonic stacks in solution. Despite the rapid motions of molecules around the stacking axis revealed by computation, relationships between molecules are fixed in separate regions of the overall potential energy surface and likely interconvert at room temperature only by scission and reformation of chains. The combined computational analyses served to evaluate two earlier proposals ${ }^{79}$ that had aimed at bringing into correspondence X-ray scattering experiments of LCLCs and the failure of Onsager's theories of isotropic to lyotropic phase transition concentration. Our computational analyses indicate that the proposed shifts or stacking faults are possible but so-called Y-junctions are not consistent with the dynamic stereochemistry of SSY aggregates. The equi-energetic configurations of SSY predicted for solution aggregates in water are matched exactly by the arrangement of molecules in the disordered crystal structure. It is as if the crystal preserves a record of the SSY condition in solution. SSY may therefore grow by the incorporation of aggregates into the crystal intact, a growth mechanism currently being explored in other systems. $^{105,106,107}$

\section{- ACKNOWLEDGMENTS}

BK thanks the US National Science Foundation (CHE-0845526, DMR-1105000) for support of this research. PR thanks the Australian Research Council for funding (FT130100463). iVEC and the National Computational Infrastructure are also gratefully acknowledged for the provision of computer time. MDW thanks the US National Science Foundation (DMR-0906576, DMR-1308677). The Bruker AXS SMART APEXII Single Crystal Diffractometer was acquired through the support of the Molecular Design Institute in the Department of Chemistry at New York University. The Bruker AXS D8 DISCOVER GADDS XRay Microdiffractometer was acquired through the support of the National Science Foundation CRIF Program (CHE-0840277) and the NSF MRSEC Program (DMR-0820341). Finally we would like to acknowledge useful discussions with Prof. J. D. Gale at Curtin University.

\section{- ASSOCIATED CONTENT}

\section{Supporting Information Available}


Key input files for the SIESTA, GULP and LAMMPS simulations in this work, along with selected output files, are provided at http://bit.ly/ $1 \mathrm{fq} 7 \mathrm{mYK}$ for reference. They are assigned the following DOI: 10.4225/06/4ED6B979EBGE6. Pages S1-S21: Crystal of $\mathrm{G}_{2} \mathrm{SSY} \cdot \mathrm{THF} \cdot 2 \mathrm{H}_{2} \mathrm{O}$ with indexed faces, NMR spectra, thermal gravimetric analysis, partial charges, interatomic potentials, and overlaid structure computed by vdW-DFT and the GAFF force-field, and crystallographic information file. This material is available free of charge via the Internet at http://pubs.acs.org.

\section{- AUTHOR INFORMATION}

\section{Corresponding Authors}

bart.kahr@nyu.edu,a.rohl@curtin.edu.au,P.Raiteri@curtin.edu.au

Notes

The authors declare no competing financial interests.

\section{- REFERENCES}

1. Sandquist, H. Ber. Dtsch. Chem. Ges. 1915, 48, 2054.

2. Lydon J. Curr Opin Colloid Interfac. Sci. 1998, 3, 458.

3. Lydon, J. Curr. Opin. Colloid Interfac. Sci. 2004, 8, 480

4. Dickinson, A. J.; LaRacuente, N. D.; McKitterick, C. B.; Collings, P. J. Mol. Cryst. Liq. Cryst. 2009, 509, 9 .

5. Collings, P. J.; Dickinson, A. J.; Smith, E. C. Liq. Cryst. 2010, 37, 701.

6. Tam-Chang, S.-W.; Huang, L. Chem. Commun. 2008, 195, 1957.

7. Park, H.-S.; Lavrentovich, O. D. Liquid Crystals Beyond Displays: Chemistry, Physics, and Applications, Li, Q. Ed. John Wiley \& Sons, New York, 2012.

8. Lydon, J. E. Mol. Cryst. Liq. Cryst. Lett. 1980, 64, 19

9. Hartshorne, N. H.; Woodard, G. D. Mol. Cryst. Liq. Cryst. 1973, 23, 343.

10. Nastishin, Y. A.; Liu, H.; Shiyanovskii, S. V.; Lavrentovich, O. D.; Kostko, A. F.; Anisimov, M. A. Phys. Rev. E, 2004, 70, 051706.

11. Nastishin, Y. A.; Liu, H.; Schneider, T.; Nazarenko, V.; Vasyuta, R.; Shiyanovskii, S. V.; Lavrentovich, O. D. Phys. Rev. E, 2005, 72, 041711.

12. Lydon, J. J. Mater. Chem. 2010, 20, 10071.

13. Dreyer, J. F. J. Phys. Colloid Chem., 1948, 52, 808.

14. Lavrentovich, M.; Sergan, T.; Kelly, J. Mol. Cryst. Liq. Cryst., 2004, 409, 21.

15. Helfinstine, S. L.; Lavrentovich, O. D.; Woolverton, C. J. Lett. Appl. Microbiol., 2006, 43, 27.

16. Colour Index, 3rd ed. Society of Dyers and Colourists, London, 1982

17. Horowitz, V. R.; Janowitz, L. A.; Modic, A. L.; Heiney, P. A.; Collings, P. J. Phys. Rev. E, 2005, 72, 041710.

18. Prasad, S. K.; Nair, G. G.; Hegde, G.; Jayalakshmi, V. J. Phys. Chem. B, 2007, 111, 9741.

19. Edwards, D. J.; Ormerod, A. P.; Tiddy, G. J. T.; Jaber, A. A.; Mahendrasingham, A. In: PhysicoChemical Principles of Colour Chemistry: Advances in Colour Chemistry Series, (Peters, A. T.; 
Freeman, H. S. Eds), Springer-Verlag, New York, 1996, vol. 4, pp. 83.

20. Tiddy, G. J. T.; Hassan, S.; Rowe, W. in Handbook of Applied Surface and Colloid Chemistry, Holmberg, K., Ed.; John Wiley \& Sons Ltd, New York, 2002; p 465.

21. Joshi, L.; Kang, S.-W.; Agra-Kooijman, D. M.; Kumar, S. Phys. Rev. E, 2009, 80, 041703.

22. Renshaw, M. P.; I. J. Day, J. Phys. Chem. B, 2010, 114, 10032.

23. Park, H.-S.; Kang, S.-W.; Tortora, L.; Kumar, S.; Lavrentovich, O. D. Langmuir, 2011, $27,4164$.

24. Kim, Y.-J.; Kim, D.-Y.; Lee, J.-H.; Nah, C.; Lee, J. H. Lee, M.-H.; Kim, H. Y.; Kuo, S.-W.; Shin, S.; Jeong, K.-U. J. Mater. Chem. 2012, 22, 13477.

25. Zhou, S.; Nastishin, Yu. A.; Omelchenko, M. M.; Tortora, L.; Nazarenko, V. G.; Boiko, O. P.; Ostapenko, T.; Hu, T.; Almasan, C. C.; Sprunt, S. N.; Gleeson, J. T.; Lavrentovich, O. D. Phys. Rev. Lett. 2012, 109, 037801.

26. Jeong, J.; Davidson, Z. S.; Collings, P. J.; Lubensky, T. C.; Yodh, A. G. Proc. Nat. Acad. Sci. 2014, 111,1742 .

27. Hamodrakas, S.; Geddes, A. J.; Sheldrick, B. J. Pharm. Pharmacol. 1974, 26, 54.

28. Chen, L. R.; Young, V. G. Jr.; Lechuga-Ballesteros, D.; Grant, D. J. W. J. Pharm. Sci. 1999, 88, 1191.

29. Stephenson, G. A.; Diseroad, B. A. Intern. J. Pharm. 2000, 198, 167.

30. Huang, L.; Tam-Chang, S.-W.; Seo, W.; Rove, K. Adv. Mater., 2007, 19, 4149.

31. Kahr, B.; Chow, J. K.; Peterson, M. L. J. Chem. Ed. 1994, 71, 584.

32. Kelley, M. P.; Janssens, B.; Kahr, B.; Vetter, W. J. Am. Chem. Soc. 1994, 116, 5519.

33. Gurney, R. W.; Mitchell, C.; Bastin, L.; Ham, S.; Kahr, B. J. Phys. Chem. B, 2000, 104, 878.

34. Carter, D.J.; Rohl, A. L.; Gale, J. D.; Fogg, A. M.; Gurney, R.W.; Kahr, B. J. Mol. Struct. 2003, 647,65 .

35. France, W. G.; Wolfe, K. M. J. Phys. Chem. 1941, 45, 395-401.

36. Rigterink, M. D.; France, W. G. J. Phys. Chem. 1938, 42, 1079-1088.

37. Kahr, B.; Jang, S.-H.; Subramony, J. A.; Bastin, L.; Kelley, M. P. Adv. Mater. 1996, 8, 941-944

38. Subramaony, J. A.; Jang, S.H.; Kahr, B. Ferroelectrics, 1997, 191, 293.

39. Hirota, S.; Miki, H.; Fukui, K.; Maeda, K. J. Cryst. Growth, 2002, 235, 541.

40. Asakuma, Y.; Ukita, E.; Maeda, K.; Fukui, K.; Iimura, K.; Suzuki, M.; Hirota, M. Cryst. Growth Des. 2007, 7, 420.

41. Velikhov, Yu.; Pritula, I.; Ganina, I.; Kolybayeva, M.; Puzikov, V.; Levchenko, A. N. Cryst. Res. Technol. 2007, 42, 27.

42. Kurimoto, M.; Müller, B.; Kaminsky, W.; Kahr, B.; Jin, L.-W. Mol. Cryst. Liq. Cryst. 2002, 389, 1.

43. Rybarska, J.; Piekarska, B.; Stopa, B.; Zemanek, G.; Konieczny, L.; Nowak, M.; Kró1, M.; Roterman, I.; Szymczakiewicz-Multanowska, A. Folia Histochem. Cytobiol. 2001, 39, 307.

44. Skowronek, M.; Roterman, I.; Konieczny, L.; Stopa, B.; Rybarska, J.; Piekarska, B.; J. Comput. Chem. 2000, 21, 656.

45. Stopa, B.; Jagusiak, A.; Konieczny, L.; Piekarska, B.; Rybarska, J.; Zemanek, G.; Król, M.; Piwowar, P.; Roterman, I. J. Mol. Model, 2013, 19, 4731.

46. Jin, L.-W.; Claborn, K.; Kurimoto, M.; Sohraby, F.; Estrada, M.; Kaminsky, W.; Kahr, B. Proc. Natl. Acad. Sci. 2003, 100, 15294.

47. Brinkworth, R. I.; Fairlie, D. P. Biochem. Biophys. Res. Commun. 1992, 188, 624.

48. Balzarini, J.; Mitsuja, H.; De Clerq, E.; Broder, S. Int. J. Cancer, 1986, 37, 451.

49. Chami, F.; Wilson, M. R. J. Am. Chem. Soc. 2010, 132, 7794-7802. 
50. Laio, A.; Parrinello, M. Proc. Natl. Acad. Sci. USA, 2002, 99, 12562-12566.

51. APEX2 (version 2013.12). Program for Bruker CCD X-ray Diffractometer Control, Bruker AXS Inc., Madison, WI, 2013.

52. Sheldrick, G. M. SHELXTL, version 6.14. Program for solution and refinement of crystal structures, Universität Göttingen, Germany, 2009.

53. Spek A. L. Acta Cryst. 2009, D65, 148.

54. GADDS and PILOT (version 2009.5). Program for Bruker CCD X-ray Diffractometer Control, Bruker AXS Inc., Madison, WI, 2009.

55. DIFFRAC ${ }^{\text {plus }}$ EVA (version 15). Software Package for Powder Diffraction. Bruker AXS Inc., Madison, WI, 2009.

56. Mercury: Visualization and analysis of crystal structures. Macrae, C. F.; Edgington, P. R.; McCabe, P.; Pidcock, E.; Shields, G. P.; Taylor, R.; Towler, M.; van de Streek, J. J. Appl. Cryst. 2006, 39, 453.

57. Soler, J. M.; Artacho, E.; Gale, J. D.; García, A.; Junquera, J.; Ordejón, P.; Sánchez-Portal, D. J. Phys: Condens. Matter, 2002, 14, 2745.

58. Lee, K.; Murray, É. D.; Kong, L.; Lundqvist, B. I.; Langreth, D. C. Phys. Rev. B. 2010, 82, 081101.

59. Troullier, N.; Martins, J. L. Phys. Rev. B, 1991, 43, 1993.

60. Boys, S. F.; Bernardi, F. Mol. Phys. 1970, 19, 553.

61. Van Duijneveldt, Frans B.; van Duijneveldt-van de Rijdt, Jeanne G. C. M.; van Lenthe, Joop H. Chem. Rev. 1994, 94, 1873.

62. Gale, J. D.; Rohl, A. L. Mol. Simulat. 2007, 33

63. Klamt, A.; Schüürmann, G. J. Chem. Soc. Perkins Trans. 1993, 2, 799.

64. Gale, J. D. Z. Krist. 2005, 220, 552.

65. Wang, J.; Wolf, R. M.; Caldwell, J. W.; Kollman, P. A.; Case, D. A. J. Comput. Chem. 2004, 25, 1157.

66. Vanquelef, E.; Simon, S.; Marquant, G.; Garcia, E.; Klimerak, G.; Delepine, J. C.; Cieplak, P.; Dupradeau, F.-Y. Nucl. Acids Res. 2011, 39, W511.

67. Åqvist, J. J. Phys. Chem. 1990, 94, 8021.

68. Mei J, Davenport J W and Fernando G W Phys. Rev. B, 1991, 43, 4653.

69. Wu, Y.; Tepper, H. L.; Voth, G. A. J. Chem. Phys. 2006, 124, 024503.

70. Plimpton, S. J. Comp. Phys. 1995, 117, 1.

71. Tribello, G. A.; Bonomi, M.; Branduardi, D.; Camilloni, C.; Bussi, G. Comput. Phys. Commun. 2014, 185, 604.

72. Barducci, A.; Bussi, G.; Parrinello, M. Phys. Rev. Lett. 2008, 100, 20603.

73. Raiteri, P.; Laio, A.; Gervasio, F. L.; Micheletti, C.; Parrinello, M. J. Phys. Chem. B 2006, 110, 3533.

74. Turner, J. E. PhD Thesis, Leeds University, 1988.

75. Ormerod, A. P. MSc and PhD Thesis, Salford University, 1994.

76. Jabber, A. A. PhD Thesis, Keele University, 1994.

77. Luoma, R. J. Ph.D. Thesis, Brandeis University, Waltham, MA, 1995.

78. Horowitz, V. R. Thesis, Swarthmore College, Swarthmore PA, 2005.

79. Park, H.-S.; Kang, S.-W.; Tortora, L.; Nastishin, Y.; Finotello, D.; Kumar, S.; Lavrentovich, O. D. J. Phys. Chem. B, 2008, 112, 16307.

80. Ojala, W. H.; Ojala, C. R.; Gleason, W. B. Antiviral Chem. Chemo. 1995, 6, 25. 
81. Ojala, W. H.; Sudbeck, E. A.; Lu, L. K.; Richardson, T. I.; Lovrien, R. E.; Gleason, W. B. J. Am. Chem. Soc. 1996, 118, 2131.

82. Ojala, W. H.; Lu, L.-K.; Alberts, K. E.; Gleason, W. B. Acta Cryst. Sect. B. 1994, B50, 684.

83. Ojala, W. H.; Gleason, W. B.; Richardson, T. I.; Lovrien, R. E. J. Am. Chem. Soc. 1996, 118, 2131.

84. Russell, V. A.; Etter, M. C.; Ward, M. D. J. Am. Chem. Soc. 1994, 116, 1941.

85. Russell, V. A.; Evans, C. C.; Li, W.; Ward, M. D. Science, 1997, 276, 575.

86. Holman, K. T.; Pivovar, A. M.; Swift, J. A.; Ward, M. D. Acc. Chem. Res. 2001, 34, 107.

87. Horner, M. J.; Holman, K. T.; Ward, M. D. J. Am. Chem. Soc. 2007, 129, 14640.

88. Soegiarto, A. C.; Comotti, A.; Ward, M. D. J. Am. Chem. Soc. 2010, 132, 14603.

89. Soegiarto, A. C.; Ward, M. D. Cryst. Growth Des. 2009, 9, 3803.

90. Burke, N. J.; Burrows, A. D.; Mahon M. F.; Teat, S. J. CrystEngComm, 2004, 6, 429.

91. Holman, K. T.; Pivovar, A. M.; Ward, M. D. Science, 2001, 294, 1907.

92. Holman, K. T.; Martin, S. T.; Parker, D. P.; Ward, M. D. J. Am. Chem. Soc. 2001, 123, 4421.

93. Horner, M. J.; Grabowski, S.; Sandstrom, K.; Holman, K. T.; Bader, M.; Ward, M. D.; Kim, W-S. ACA Trans. 2004, 39, 1.

94. Kim, J.; Yi, J.; Ward, M. D.; Kim, W.-S. Sep. Purif. Tech. 2009, 66, 57.

95. Edwards, D. J.; Jones, J. W.; Lozman, O.; Ormerod, A. P.; Sintyureva, M.; Tiddy, G. J. T. J. Phys. Chem. B, 2008, 112, 14628.

96. Yao, X. PhD Dissertation, Georgia Institute of Technology, 2011.

97. Guerra, G.; Cavallo, L.; Corradini, P. Top. Stereochem. 2003, 24, 1.

98. Laio, A.; Gervasio, F. L. Rep. Prog. Phys. 2008, 71, 126601.

99. MacKintosh, F. C.; Safran, S. A.; Pincus, P. A. Europhys. Lett. 1990, 12, 697-702,

100. Onsager, L. Ann. N. Y. Acad. Sci. 1949, 51, 627-659.

101. Bolhius, P.; Frenkel, D. J. Chem. Phys. 1997, 106, 666-687.

102. Vroege, G. J.; Lekkerkerker, H. N. W. Rep. Prog. Phys. 1992, 55, 1241.

103. Tomasik, M. R.; Collings, P. J. J. Phys. Chem. B, 2008, 112, 9883.

104. Nastishin, Yu. A.; Liu, H.; Schneider, T.; Nazarenko, V.; Vasyuta, R.; Shiyanovskii, S. V.; Lavrentovich, O. D. Phys. Rev. E, 2005, 72, 041711.

105. Saito, A.; Igarashi, K.; Azuma, M.; Ooshima, H. J. Chem. Eng. Jpn. 2002, 35, 1133.

106. Lohani, S.; Nesmelova, I. V.; Suryanarayanan, R.; Grant, D. J. W. Cryst. Growth Des. 2011, 11, 2368.

107. Hunter, C. A.; McCabe, J. F.; Spitaleri, A. CrystEngComm, 2012, 14, 7115. 
For Table of Contents Use Only

\section{Structural Correspondence of Solution, Liquid Crystal, and Crystalline Phases of the Chromonic Mesogen Sunset Yellow}

Wenchang Xiao ${ }^{\dagger}$, Chunhua $\mathrm{Hu}^{\dagger}$, Damien J. Carter ${ }^{\ddagger}$, Shane Nichols ${ }^{\dagger}$, Michael D. Ward ${ }^{\dagger}$, Paolo Raiteri $i^{*}$, Andrew L. Rohl $l^{\ddagger^{*}}$, Bart Kahr ${ }^{\dagger^{*}}$

Table of Contents Graphic (1.375" × 1.375") 


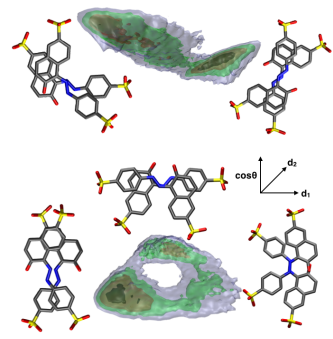

Synopsis (60 words)

A single crystal structure of sunset yellow shows a remarkable correspondence with the stereochemistry of molecules in solution and in the liquid crystal phase as established by molecular dynamics, metadynamics, and quantum chemical computations. The solution stereochemistry and stereodynamics has implications for the geometry of long rods, for which understanding is a prerequisite for reckoning properties of vexing chromonic mesophases. 